\title{
La comunicación en México: un análisis de la producción científica disciplinaria en el contexto iberoamericano*
}

\author{
Communication in Mexico: An Analysis of the Disciplinary Scientific Production in the Ibero-American Context \\ A comunicação no México: análise da produção científica disciplinar no contexto ibero-americano
}

Daniel Francisco Flores-García Universidad Autónoma del Estado de México, México ORCID: https://orcid.org/0000-0002-3292-8490

DOI: https://doi.org/10.11144/Javeriana.syp39-76.cmap

\author{
Arianna Becerril-García ${ }^{\text {a }}$ \\ Universidad Autónoma del Estado de México, México \\ arianna.becerril@gmail.com \\ ORCID: https://orcid.org/0000-0003-0278-8295
}

Recibido: 27 Abril 2020

Aceptado: 21 Septiembre 2020

Publicado: 23 Diciembre 2020

\section{Resumen:}

Se analiza la disciplina de comunicación en Iberoamérica en lo relativo a sus dinámicas de publicación y colaboración, identificando las comunidades que han participado en su narrativa científica. Se enfatiza el desempeño de México, en contexto con los principales países que han aportado a la consolidación de la comunicación como campo de conocimiento. El universo de estudio lo constituyen 8989 artículos científicos publicados entre 2005 y 2018 en las 27 revistas de comunicación integradas a Redalyc y se recurre a su metodología e indicadores para la evaluación de la producción científica. El análisis identifica la relevancia de las revistas regionales en la consolidación de la disciplina, así como la centralidad de España, Brasil y México, en la aportación de literatura científica. Además, registra la presencia de redes de colaboración primordialmente institucional, mientras que en el ámbito internacional resalta el fuerte vínculo entre Latinoamérica y España.

Palabras clave: comunicación, bibliometría, México, revistas científicas, Redalyc.

\section{Abstract:}

This work analyzes the communication discipline in Ibero-America regarding the dynamics of publication and collaboration, thus identifying the communities that have took part in its scientific narrative. It emphasizes the endeavor carried out in Mexico, in the context of the main countries that have contributed to consolidate the communication as a field of knowledge. The study universe consists of 8,989 scientific articles published between 2005 and 2018 in the 27 communication journals linked to Redalyc and are evaluated as a scientific product based on their methodology and indicators. The analysis identified the relevance of regional journals in the consolidation of this discipline as well as the central position of Spain, Brazil and Mexico thanks to their contribution to the scientific literature. In addition, this work highlights the presence of collaborator networks, which are mostly institutional, while in the international sphere it highlights the strong link between Latin America and Spain.

Keywords: communication, bibliometrics, Mexico, scientific journals, Redalyc.

\section{Resumo:}

Analisa-se a disciplina de comunicação na Ibero-América em relação à sua dinâmica de publicação e colaboração, identificando as comunidades que participaram na sua narrativa científica. Destaca-se a atuação do México no contexto dos principais países que contribuíram para a consolidação da comunicação como campo de conhecimento. O universo de estudo é constituído por 8989 artigos científicos publicados entre 2005 e 2018 nas 27 revistas de comunicação integradas na Redalyc e sua metodologia e indicadores são usados para avaliar a produção científica. A análise identifica a relevância das revistas regionais na consolidação da disciplina, bem como a centralidade da Espanha, Brasil e México, na contribuição da literatura científica. Além disso, registra a presencia de redes de colaboração principalmente institucionais, enquanto no âmbito internacional destaca a forte ligação entre a América Latina e a Espanha.

Palavras-chave: comunicação, bibliometria, México, revistas científicas, Redalyc.

Notas de autor

\footnotetext{
a Autor de correspondencia. Correo electrónico: arianna.becerril@gmail.com
} 


\section{Introducción}

La comunicación, vista como comunidad, se ha mantenido en constante evolución en busca de consolidar un campo de conocimiento que ofrezca distintas formas teóricas y metodológicas para abordar los problemas de su área de estudio, generando recursos intelectuales diversos para consolidarla como una disciplina práctica y un medio semiótico con fines sociales (Jensen, 1995 citado en Craig, 1999, 2013; Fuentes-Navarro, 2010a). De tal modo, la importancia de esta disciplina radica en que se alimenta y comparte su objeto de estudio con otros campos disciplinares con el objetivo de estudiar, desde enfoques diversos, los procesos de producción, circulación, intercambio, negociación y uso de las formas simbólicas en la esfera social (Méndez-Rátiva \& Gregorio-Chaviano, 2014; Pereira, 2005).

Pese al permanente proceso de construcción y consolidación de la disciplina, en el sentido de la "gran conversación", como lo plantea Jean Claude Guédon (2017), las ciencias sociales, y en específico la comunicación, no están representadas de la misma forma en las diferentes bases de datos, ya sea comerciales o aquellas en acceso abierto, las cuales se han consolidado en los ámbitos "naturales" de difusión y visibilidad, tanto en América Latina como en el Norte global.

Por ejemplo, en el SCImago Journal \& Country Rank (SJR) edición 2019, y con información de Scopus, la producción científica de ciencias sociales representó el 20,4\%, en contraste, con la base de datos de Redalyc, donde el 50,5\% de los artículos pertenecen a ciencias sociales. Una representatividad similar se observa en la producción científica de la disciplina de comunicación en ambos universos: en el SJR representa el 0,4\% del total de artículos, mientras que en Redalyc representa el $2 \%$, presencia que, si bien es menor, quintuplica el peso de la primera base en lo relativo (Figura 1). Tomando como ejemplo ambas bases datos, una de ellas característica del mainstream de la ciencia y la otra de corte regional, se observan dos panoramas distintos en lo referente a la representatividad de las ciencias sociales y la comunicación. A su vez, cabe resaltar que, en el universo de información provisto por Redalyc, el 93,8 \% de los artículos de la comunicación corresponde a autores adscritos a Iberoamérica. Así, se trata de una diferencia de representatividad no solo disciplinar, sino geográfica, lo que en conjunto afecta las condiciones de visibilidad, legitimidad y posibilidades de construcción de comunidad.
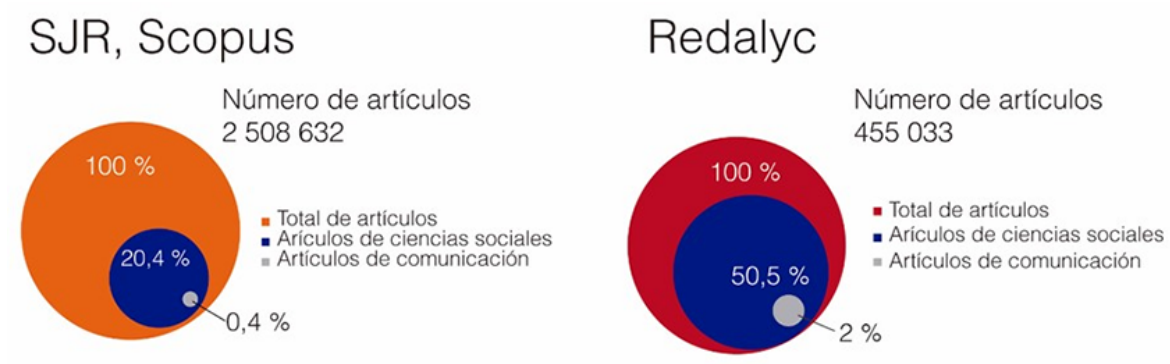

FIGURA 1.

Comparativo entre las bases de datos de Scopus y Redalyc.

Fuente: elaboración propia a partir de datos de SCImago Journal \& Country Rank (2019) y Redalyc (2020).

Cabe preguntarse, ¿Por qué en ambas bases, pero particularmente en Redalyc que adscribe principalmente a revistas de ciencias sociales, la disciplina de comunicación tiene baja presencia? Podemos aventurar que obedece a la juventud de la disciplina. Al respecto, Soto del Ángel (2008), retomando a Jesús Galindo y a Raúl Fuentes Navarro, distingue tres modelos fundacionales en la institucionalización de la comunicación como campo académico en México y en América Latina: la formación de periodistas, el comunicador como intelectual y el comunicólogo, atribuyendo el modelo del comunicador como intelectual a la Universidad Iberoamericana (México), en 1960, con la institucionalización de la primera Licenciatura de Comunicación. A su vez, en la institucionalización de la Comunicación como campo científico, Fuentes Navarro (2010b) 
destaca el papel del Centro Internacional de Estudios Superiores de Comunicación para América Latina (CIESPAL), en la conversión de las escuelas latinoamericanas de periodismo en escuelas de comunicación en los años sesenta, donde las universidades privadas tomaron el liderazgo.

Con poco más de medio siglo, la trayectoria de la comunicación como disciplina se refleja en la existencia de órganos de difusión más jóvenes, lo que puede ejemplificarse con la trayectoria de la Revista Mexicana de Sociología, la cual surge en 1939, veinte años antes de la primera licenciatura de Comunicación en la Universidad Iberoamericana, México. Una segunda conjetura que puede plantearse respecto a la falta de representatividad de la comunicación en Redalyc y, en general, en las bases de datos, es que, debido a su juventud, sus mecanismos de construcción de comunidad y legitimidad descansaron en otros valores y no en la necesidad de indizar sus órganos de difusión. Siguiendo con el ejemplo de la sociología, y observando el Directorio de Latindex, uno de los sistemas científicos con mayor cobertura de la ciencia regional: existen 456 títulos únicos de revistas de ciencias de comunicación en Iberoamérica, mientras que de sociología se tiene registro de 1043 títulos únicos.

A pesar de que Latinoamérica y México son actores significativos en la producción y consolidación de ciencias sociales regionales, como es el caso de la disciplina de comunicación, no existen trabajos que tracen su trayectoria o caractericen la producción científica disciplinar. En su lugar, los estudios bibliométricos sobre la disciplina de comunicación presentan sesgos idiomáticos, disciplinares y geográficos, ya que se basan principalmente en las bases de datos de la Web of Science (WoS) y Scopus, cuyo universo y métricas (Factor de impacto o Índice H) muestran sesgos ampliamente documentados; ejemplo de ello son los trabajos de Méndez-Rátiva y Gregorio-Chaviano (2014), Castillo-Esparcia et al. (2012) y Rodríguez (2010).

En atención a lo anterior, el presente trabajo se plantea como objetivo caracterizar la producción iberoamericana de la disciplina de comunicación publicada entre 2005 y 2018, en revistas científicas de acceso abierto, tomando como universo de información las integradas a Redalyc. En el análisis, se hace énfasis en la producción científica de la comunidad mexicana, observando las dinámicas de publicación de las comunidades científicas de cada país, así como de las prácticas y tipos de colaboración científica bajo las cuales se ha generado tal producción académica. En ello, se recurre a la metodología e indicadores propuestos por Redalyc, los cuales parten de una perspectiva que busca dar cuenta de las dinámicas y prácticas de las comunidades científicas, para poder trazar una cartografía de la forma en que las diversas comunidades científicas aportan a la narrativa científica; a su vez, se parte de un enfoque retrospectivo en el análisis de la disciplina de comunicación a partir de su producción científica, y la caracterización se hace en términos cuantitativos, en la identificación de tendencias y cualitativos, en el reconocimiento de los actores que aportan a la construcción de la comunicación como campo de conocimiento y en la interpretación de los resultados.

La particularidad del presente análisis radica en que se realiza una caracterización de la producción científica de comunicación en acceso abierto, un universo poco documentado, pero de gran relevancia para áreas de conocimiento como las ciencias sociales y para regiones como América Latina, región cuyo modelo de comunicación científica tiene como base un acceso abierto no comercial (sin cobros por publicar en revistas científicas ni por acceder a la literatura científica): de acuerdo con información de DOAJ (Directory of Open Access Journals), uno de los universos editoriales más incluyentes a nivel global, entre las 2530 revistas científicas latinoamericanas incluidas, menos del $5 \%$ cobran al autor por publicación (APC, articleprocessing charge) y del $100 \%$ que lo hace, el 73 \% proviene de Brasil (Aguado-López \& Becerril García, 2019). Así, el presente artículo da cuenta de producción científica que fue comunicada a las comunidades académicas y a la sociedad en general, bajo un esquema de publicación abierto a autores y que se encuentra disponible para su libre consulta. 


\section{Método y datos}

El análisis se basa en un universo de información constituido por 8.989 artículos científicos publicados entre 2005 y 2018, en las 27 revistas de comunicación integradas a Redalyc hasta diciembre de $2018^{[1]}$. El núcleo de información que se analiza es el resultado del trabajo de 13.672 formas de autor, con adscripción a 1.308 instituciones de 65 países. En ello, se hace énfasis en las dinámicas de publicación y colaboración de la comunidad mexicana, cuya producción científica son 1.053 artículos publicados en el mismo periodo, por 1.435 formas de autor con adscripción a 116 instituciones y publicada en 25 revistas científicas. La composición del universo de estudio se especifica en la Figura 2. Con la finalidad de trazar una cartografía de la disciplina basada en las contribuciones de carácter científico, del análisis se excluyeron documentos como editoriales, entrevistas, reseñas y demás escritos que no hubieran sido sometidos a una revisión por pares ciegos.

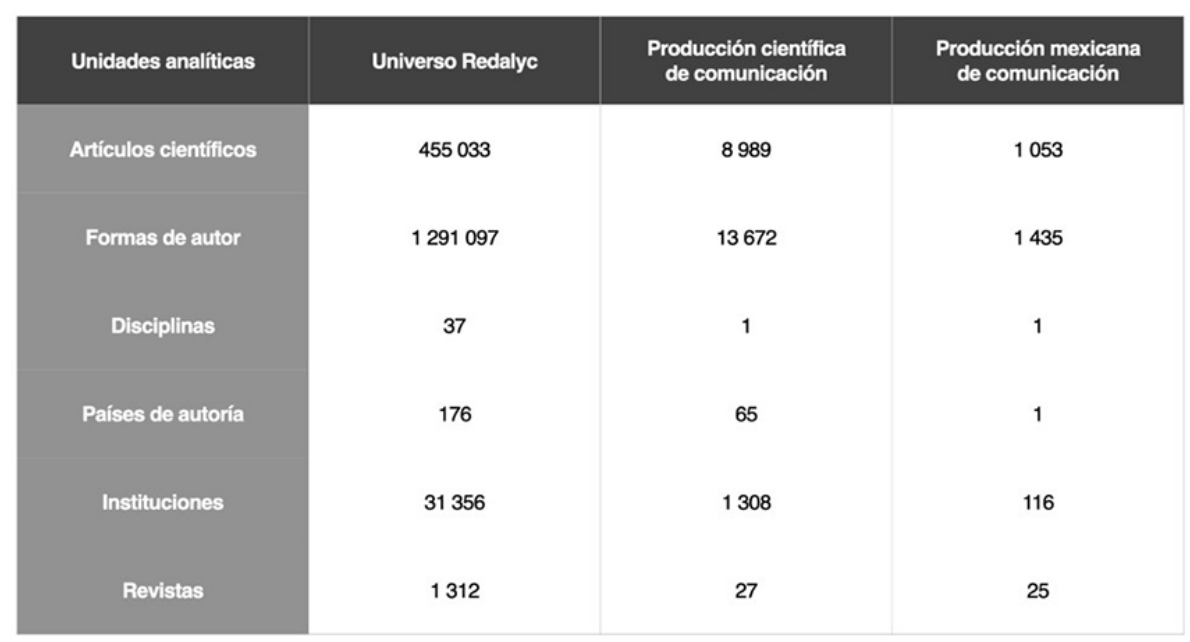

FIGURA 2.

Constitución del universo de estudio, 2005-2018

Fuente: elaboración propia a partir de datos de Redalyc, UAEM (2020).

El análisis se orientó con base en la metodología e indicadores propuestos por Redalyc, los cuales, entre otras variables, permiten dar cuenta de las prácticas y tendencias de publicación y colaboración, permitiendo identificar la procedencia y peso de la publicación de los distintos actores que participan en el quehacer científico. La metodología e indicadores propuestos parten de una perspectiva analítica que permite informar sobre las prácticas específicas que están dando forma al conocimiento científico regional y desde un universo representativo en lo disciplinar, lo regional y lo idiomático (Aguado-López et al., 2013; López Castañares et al., 2013). Como se observa en la Figura 3, los indicadores usados pueden partir de siete entidades: revistas, artículos, áreas de conocimiento, disciplinas, países, autores e instituciones. En el caso del presente análisis, la entidad que se aborda es la disciplina de comunicación, y en ello se realiza una diferenciación de tendencias por país. Los indicadores de la metodología Redalyc de los cuales se hace uso son:

1. Producción: es la variable general que hace referencia a la publicación científica, la cual puede ser vista desde el origen de la producción científica o desde el origen de la producción editorial. Con la finalidad de distinguir con precisión las dinámicas de publicación, esta variable de cataloga a su vez en:

a) Producción externa: se trata de artículos científicos publicados en un país distinto al país de adscripción del autor. En esta categoría se identifican dos componentes: 
i. Producción de fuera de Latinoamérica: se trata de la producción científica publicada en países no latinoamericanos. En el caso del presente estudio, esta variable se limita a identificar la producción publicada en España, puesto que es la única región distinta a Latinoamérica considerada en el universo de estudio, ya que en la cobertura de Redalyc las revistas de la disciplina de Comunicación provienen únicamente de países de Latinoamérica y de España (Tabla 1).

ii. Producción de Latinoamérica: se trata de la producción publicada en países latinoamericanos, ajenos al de adscripción del autor.

b) Producción interna: se trata de artículos científicos publicados en revistas que pertenecen al mismo país de adscripción del autor. Esta variable se compone a su vez de dos categorías:

i. Producción nacional: hace referencia a los artículos científicos publicados en revistas editadas en el mismo país del autor, pero de institución distinta.

ii. Producción institucional: hacer referencia a los artículos científicos publicados en revistas editadas por la misma institución de adscripción del autor.

2. Colaboración:

a) Producción en colaboración: se trata de la producción científica publicada en coautoría. Se considera a esta variable como principal evidencia empírica para dar cuenta del fenómeno de colaboración científica. Esta variable se subdivide en:

i. Colaboración externa: se trata de producción científica publicada en coautoría y cuyos autores tienen adscripción a países distintos. A su vez, este componente se constituye por:

1. Colaboración fuera de Latinoamérica: hace referencia a las redes de colaboración construidas a partir de la coautoría entre autores de países distintos y fuera de Latinoamérica.

2. Colaboración con Latinoamérica: redes de colaboración construidas a partir de la coautoría entre autores adscritos a Latinoamérica y autores adscritos a países no latinoamericanos. En el presente estudio, se hace referencia a esta variable como "Latam y otras regiones".

3. Colaboración en Latinoamérica: redes de colaboración construidas a partir de la coautoría entre autores adscritos a países latinoamericanos diferentes.

ii. Colaboración interna: se trata de producción científica publicada en coautoría y cuyos autores comparten adscripción por país.

1. Colaboración nacional: colaboración entre autores adscritos al mismo país, pero a instituciones distintas.

2. Colaboración institucional: colaboración entre autores que comparten adscripción institucional.

b) Producción sin colaboración: se trata de la producción científica publicada por un único autor. 


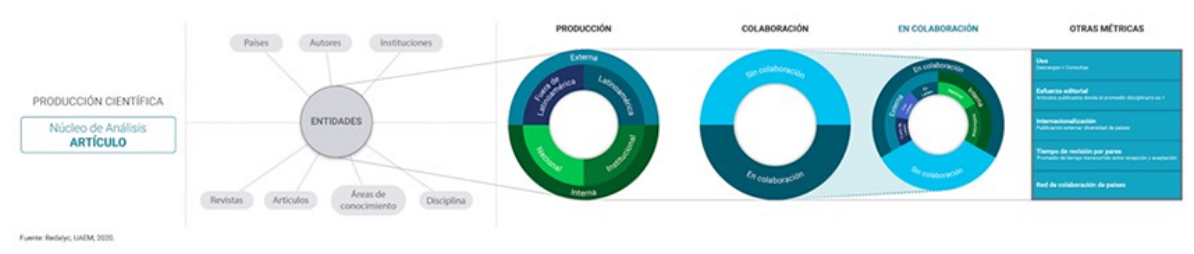

FIGURA 3.

Metodología e indicadores de $2^{\circ}$ generación de Redalyc

Fuente: elaboración propia a partir de datos de Redalyc, UAEM (2020)

\section{Resultados}

La disciplina de comunicación ha construido una comunidad de 13672 formas de autor con adscripción a 1308 instituciones de 65 países que han generado 8989 artículos científicos (9341 si se les desagrega por país). En la Figura 4, se representa la distribución de producción científica y formas de autor ${ }^{[2]}$ por país en el periodo 2005-2018, donde destaca la participación central de España, Brasil y México, países pilares en la consolidación de la disciplina, lo que se refleja en que concentran el 68,2 \% de la producción y el 70,4 \% de las formas de autor. Resalta la diversidad de países con producción científica en la disciplina, donde, a la participación central de América Latina y España, se suman regiones como Europa, Norteamérica, Asia y África.

Cabe destacar que la comunidad mexicana de la comunicación asciende a 1435 formas de autor, las cuales han generado un total de 1053 artículos científicos que han sido publicados en 26 de las 27 revistas de la disciplina, lo que la posiciona como la tercera comunidad nacional más grande a nivel internacional, pese a que se trata de una disciplina joven, especialmente en el país, como se abordó en el apartado anterior del presente escrito. Un segundo aspecto por destacar es que ha publicado en 26 de las 27 revistas del área incluidas en Redalyc, lo que habla del dinamismo y diversificación del diálogo propuesto por dicha comunidad.

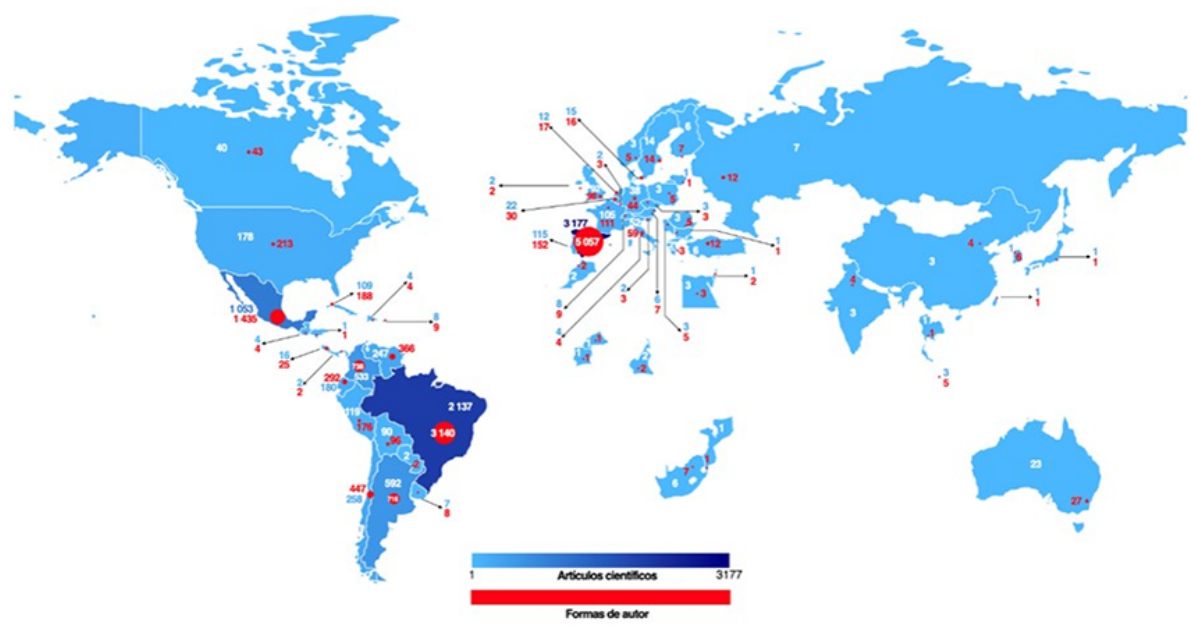

FIGURA 4.

Países, formas de autor y producción científica por país en la disciplina de Comunicación, 2005-2018 Fuente: elaboración propia a partir de datos de Redalyc, UAEM (2020).

Entre los 1308 centros de investigación que han realizado aportaciones a la disciplina, destacan principalmente 20, los cuales concentran el 29,4 \% de la producción. Resaltan instituciones centrales españolas, mexicanas y brasileñas como principales nodos de generación de conocimiento en la disciplina 
(Figura 5). En el caso de las instituciones mexicanas, destacan el Instituto Tecnológico y de Estudios Superiores de Monterrey, la Universidad Nacional Autónoma de México y la Universidad Autónoma Metropolitana, como centros nacionales que han hecho la mayor cantidad de aportes científicos a la disciplina.

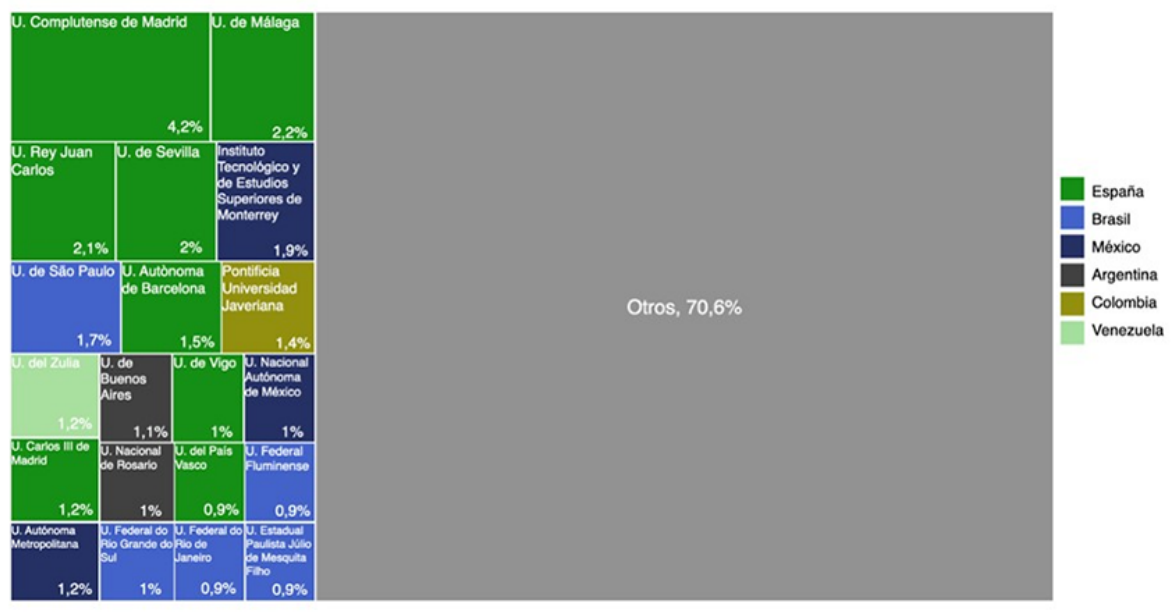

FIGURA 5.

Aporte por institución a la producción científica de la disciplina de Comunicación, 2005-2018 Fuente: elaboración propia a partir de datos de Redalyc, UAEM (2020).

La comunidad mexicana de la comunicación muestra una tendencia de publicación principalmente endógena (65,9\% de la publicación), dado que el 48,8 \% de su producción ha sido publicada en revistas nacionales y el $17,1 \%$ en revistas institucionales. Se trata de una tendencia que, no obstante, comienza a invertirse a partir de 2014, al ir ganando mayor recurrencia la publicación en revistas de otros países, principalmente de Latinoamérica (Figura 6). En la dinámica de publicación de la disciplina en general, resalta la recurrencia de publicación en las revistas latinoamericanas, la cual tiene un peso relativo significativo en cada uno de los principales países que mayor producción han aportado, lo que denota que la comunicación se ha consolidado en buena medida dado al intercambio y debate que ha tenido lugar en las revistas regionales. Sin embargo, cabe llamar la atención del peso que tiene la publicación en España, el cual, en algunos casos, tiene uno similar a la de revistas regionales, como es el caso de México, Ecuador y Perú. La importancia de señalar esta tendencia radica en que algunos países están priorizando la publicación en un solo país, por encima de los diferentes países de Latinoamérica, lo que tiene implicaciones en el tipo y orientación de la narrativa que se está construyendo en la disciplina. 


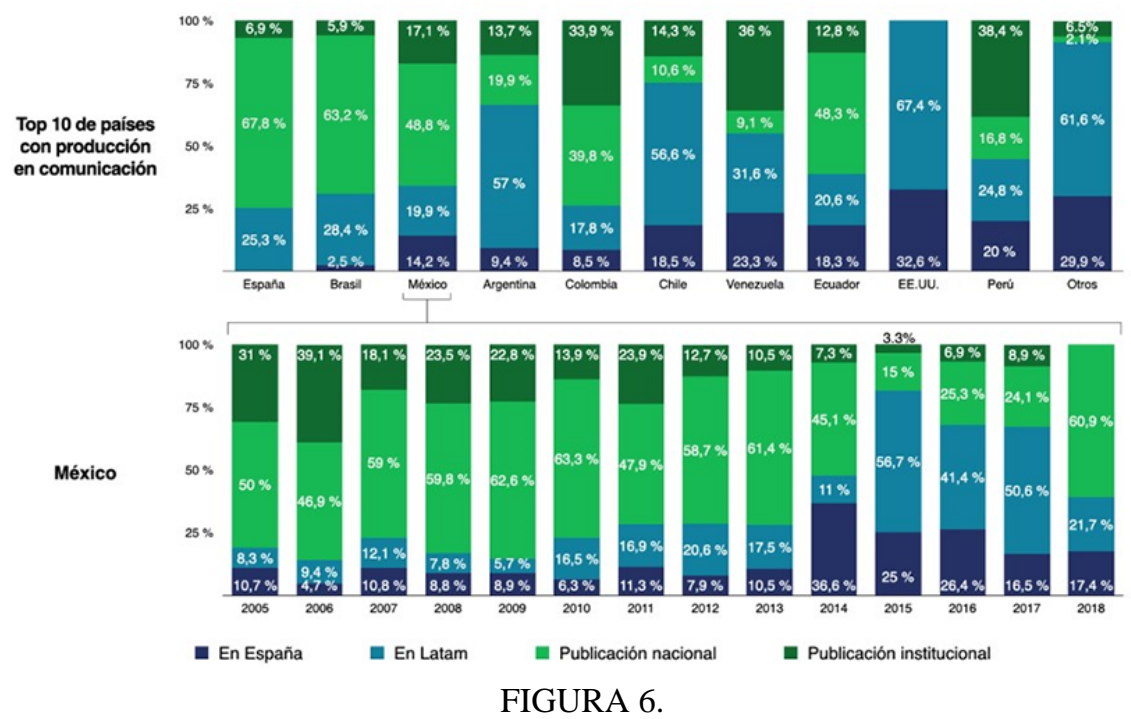

Tipos de publicación en la disciplina de Comunicación, tendencias por país, 2005-2018 Fuente: elaboración propia a partir de datos de Redalyc, UAEM (2020).

En la Figura 7, se representa A) el potencial que cada comunidad nacional tiene para establecer un diálogo global a partir de la publicación en otros países y B) el reconocimiento internacional que cada país tiene en términos editoriales. En lo referente al primer punto, cabe destacar que las tres principales comunidades que mayor literatura académica han aportado a la disciplina son también los países que más aportan a la publicación externa: España firma el 21,2 \% de la producción externa, Brasil el 17,5 \%, México el 9,5 \%, y a ellos se suman Argentina, que aporta el 10,4\% en este rubro. En lo relativo a los países que mayor producción externa reciben, resalta que México (29\%), España (20,8 \%) y Ecuador (17,9\%) son los países con mayor posicionamiento como países editoriales en el ámbito internacional al interior de la disciplina; en este aspecto, es notoria la ausencia de Brasil como país editor de publicaciones extranjeras, lo que se explica por su alta tendencia de publicación nacional. Véase la Figura 7, donde se representa el flujo de la publicación externa en la comunicación, desde la perspectiva de la procedencia y el país editorial, con nivel de detalle de cada país. 


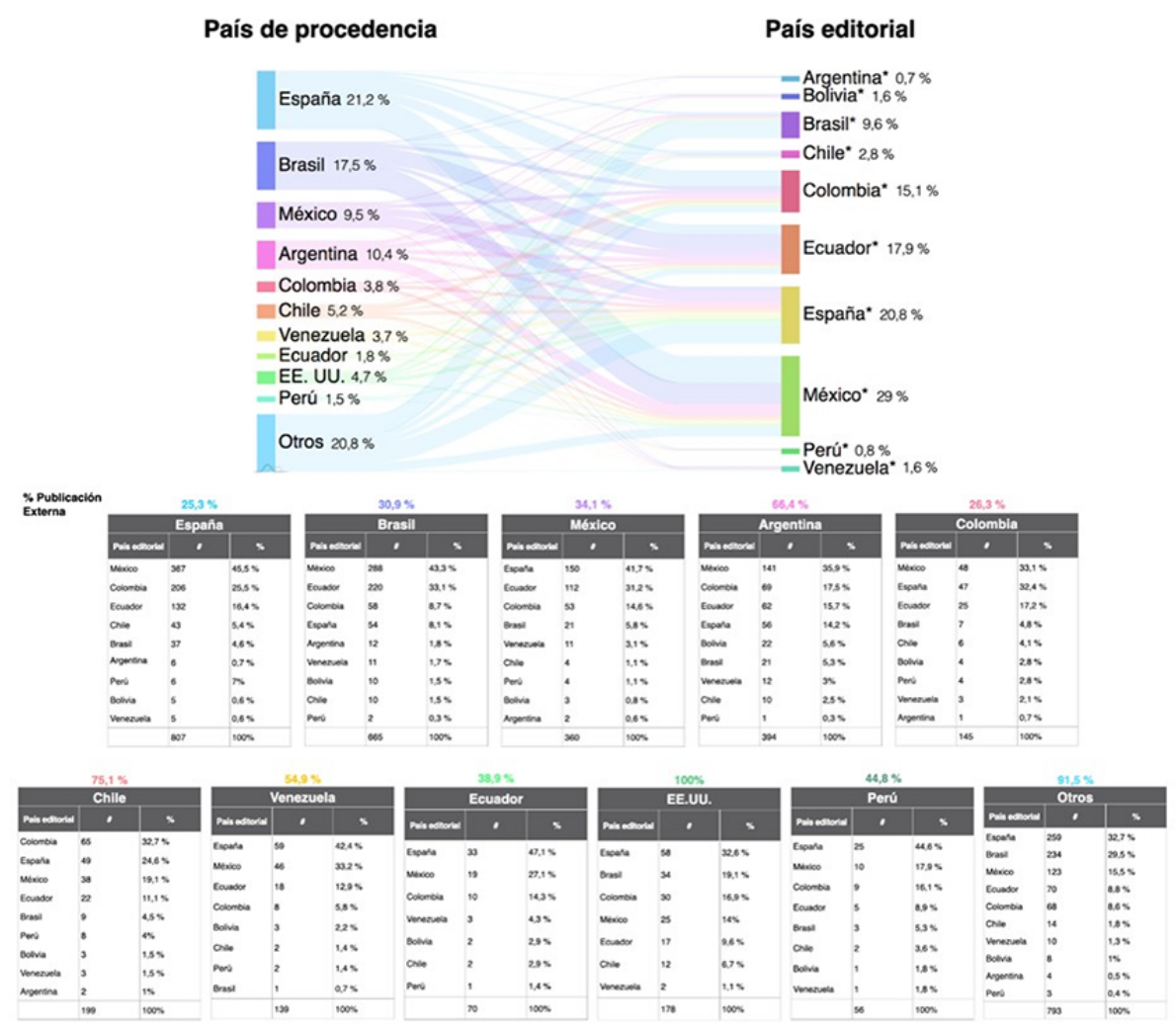

FIGURA 7.

Destino de la Publicación Externa por país en la disciplina de Comunicación, 2005-2018. Fuente: elaboración propia a partir de datos de Redalyc, UAEM (2020).

Desde otra perspectiva, en las dinámicas de generación de conocimiento en la disciplina de comunicación, la coautoría se muestra como una tendencia creciente. Como se observa en la Figura 8, la coautoría en la comunidad mexicana es gradualmente más recurrente con el paso del tiempo, dando como resultado que el $28,7 \%$ de la producción mexicana del área sea resultado de trabajo colaborativo. La adopción de esquemas colaborativos que se observa en la disciplina, se registra de forma general en todas las ciencias sociales y humanidades de Latinoamérica (Aguado-López et al., 2018; González Alcaide \& Gómez Ferri, 2014; Russell et al., 2007). 

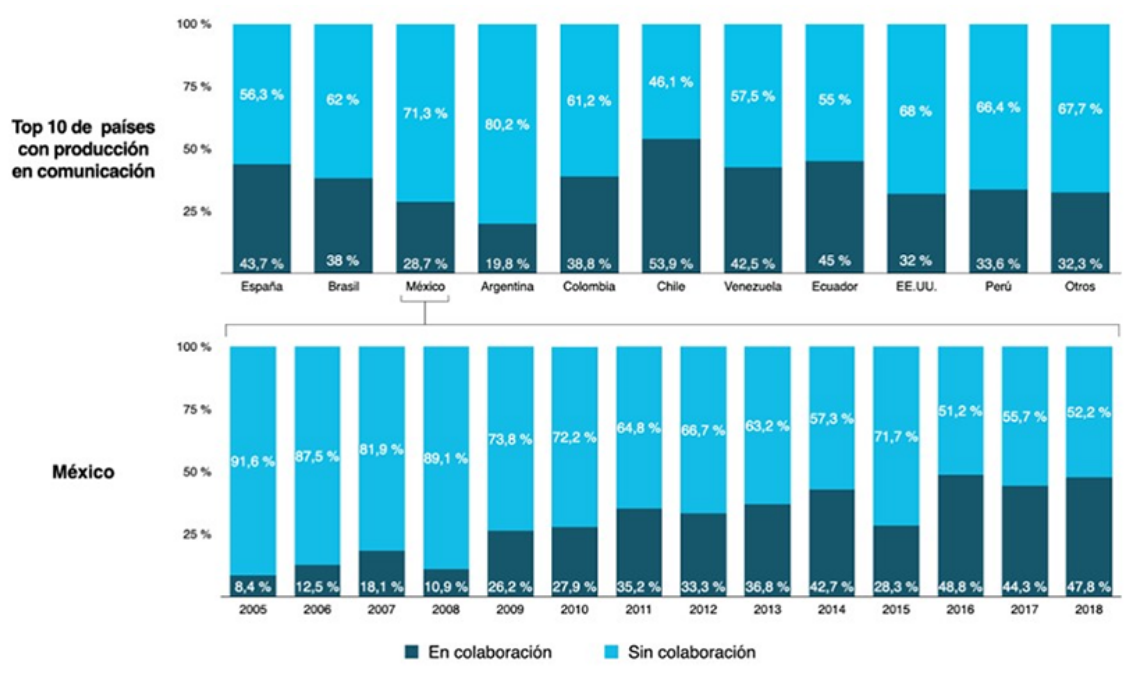

FIGURA 8.

Producción en coautoría y sin coautoría en la disciplina de Comunicación, tendencias por país, 2005-2018 Fuente: elaboración propia a partir de datos de Redalyc, UAEM (2020).

Las redes de colaboración construidas por la comunidad mexicana son principalmente de alcance institucional, como se representa en la Figura 9, mientras que la colaboración de tipo nacional se ubica como el segundo tipo de colaboración más recurrido. Esta es una tendencia que siguen en general todos los países que han participado en la narrativa de la disciplina, donde, no obstante, la colaboración externa tiene un peso significativo, principalmente las redes de colaboración entre América Latina y otras regiones, por encima de la colaboración entre países latinoamericanos y aquella donde no participa Latinoamérica.

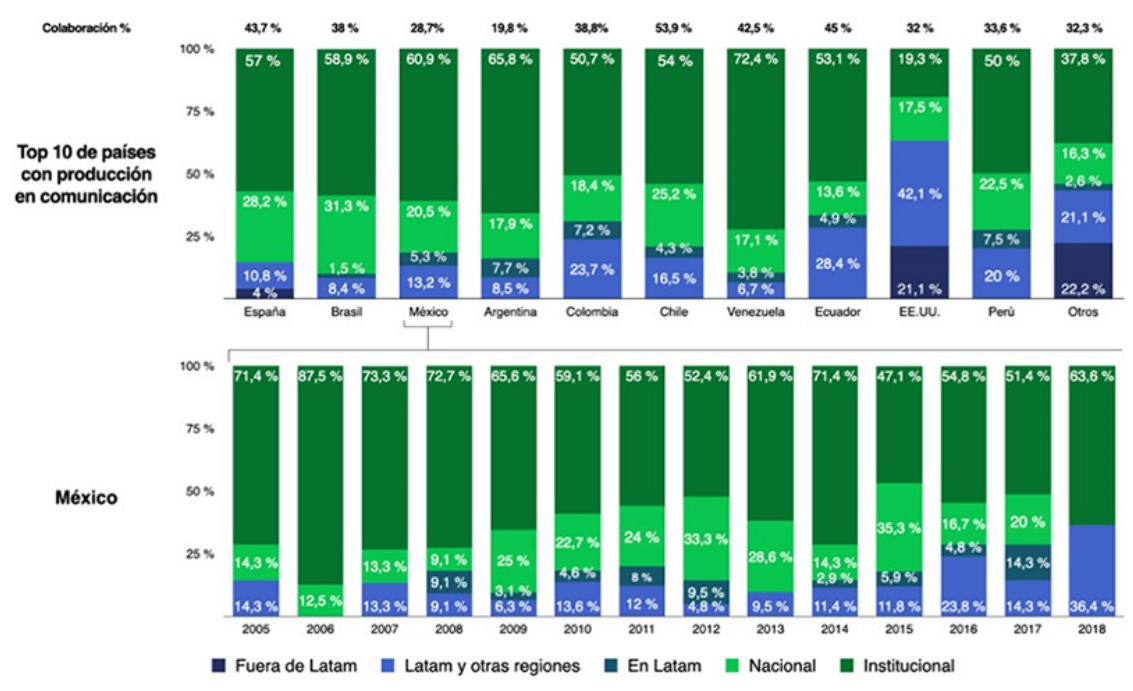

FIGURA 9.

Tipos de Colaboración en la disciplina de Comunicación, tendencias por país, 2005-2018 Fuente: elaboración propia a partir de datos de Redalyc, UAEM (2020).

En las redes de colaboración científica al interior de Latinoamérica han participado 10 países, y en ello México, Colombia y Brasil resaltan como los países que más interacciones han establecido con los demás países de la región. En específico, México ha interactuado con ocho países y esta colaboración se ha dado de manera diversificada. En Figura 10 se representan las redes de colaboración científica externa en la disciplina al interior de Latinoamérica, con detalle en las interacciones establecidas por México, en los cuales se desagrega por país. 

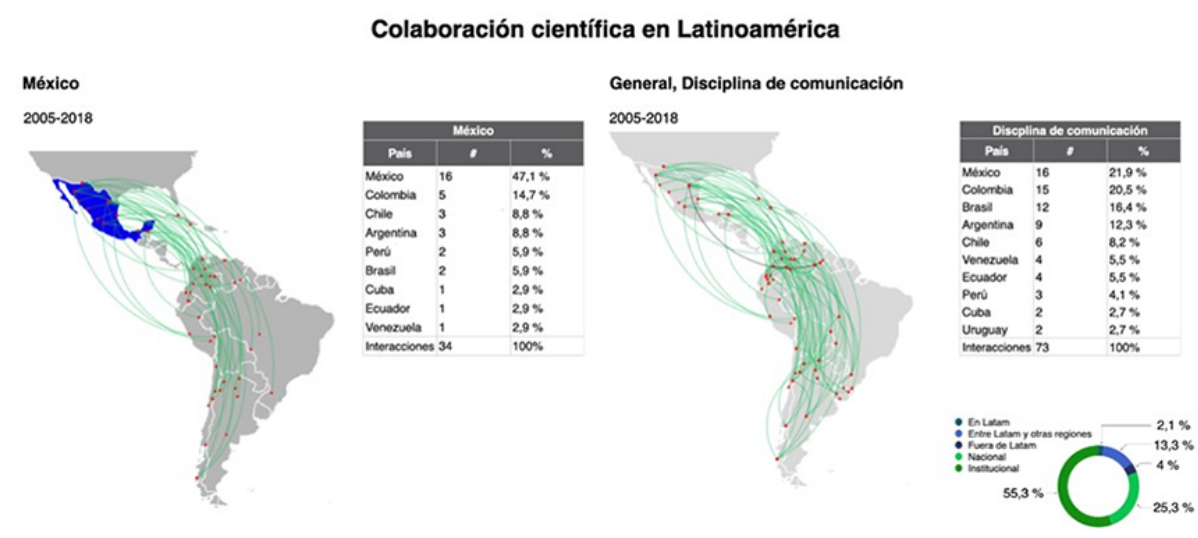

FIGURA 10.

Redes de colaboración científica en Latinoamérica, disciplina de Comunicación, 2005-2018 Fuente: elaboración propia a partir de datos de Redalyc, UAEM (2020).

En torno a las redes de colaboración construidas entre países latinoamericanos y otras regiones, se registra la participación de académicos de 25 países, lo que la hace el tipo de colaboración externa más diversificado. Asimismo, la colaboración entre Latinoamérica y otras regiones tiene un peso relativo (13,3 $\%)$ que supera seis veces el de la colaboración externa latinoamericana (2,1\%), y en ello la colaboración se ha concentrado en España. Esta distribución pone de manifiesto que la dinámica de colaboración se ha establecido principalmente entre Latinoamérica y España, como se muestra en la Figura 11, donde se registra que España concentra el 32,1 \% de las interacciones, seguido de Brasil (14,5 \%), Colombia (10,5\%) y México (8,5 \%). En el caso de la comunidad mexicana, cabe señalar que, replicando la tendencia general de la disciplina, el país con el que principalmente ha construido relaciones es España, y en casos aislados con Bélgica, Canadá, Suecia, Suiza y los Países Bajos. Este aspecto expone la centralidad de la relación entre México y España, no solamente en términos de publicación externa como se abordó en apartados anteriores, sino de colaboración científica a partir de la coautoría. 


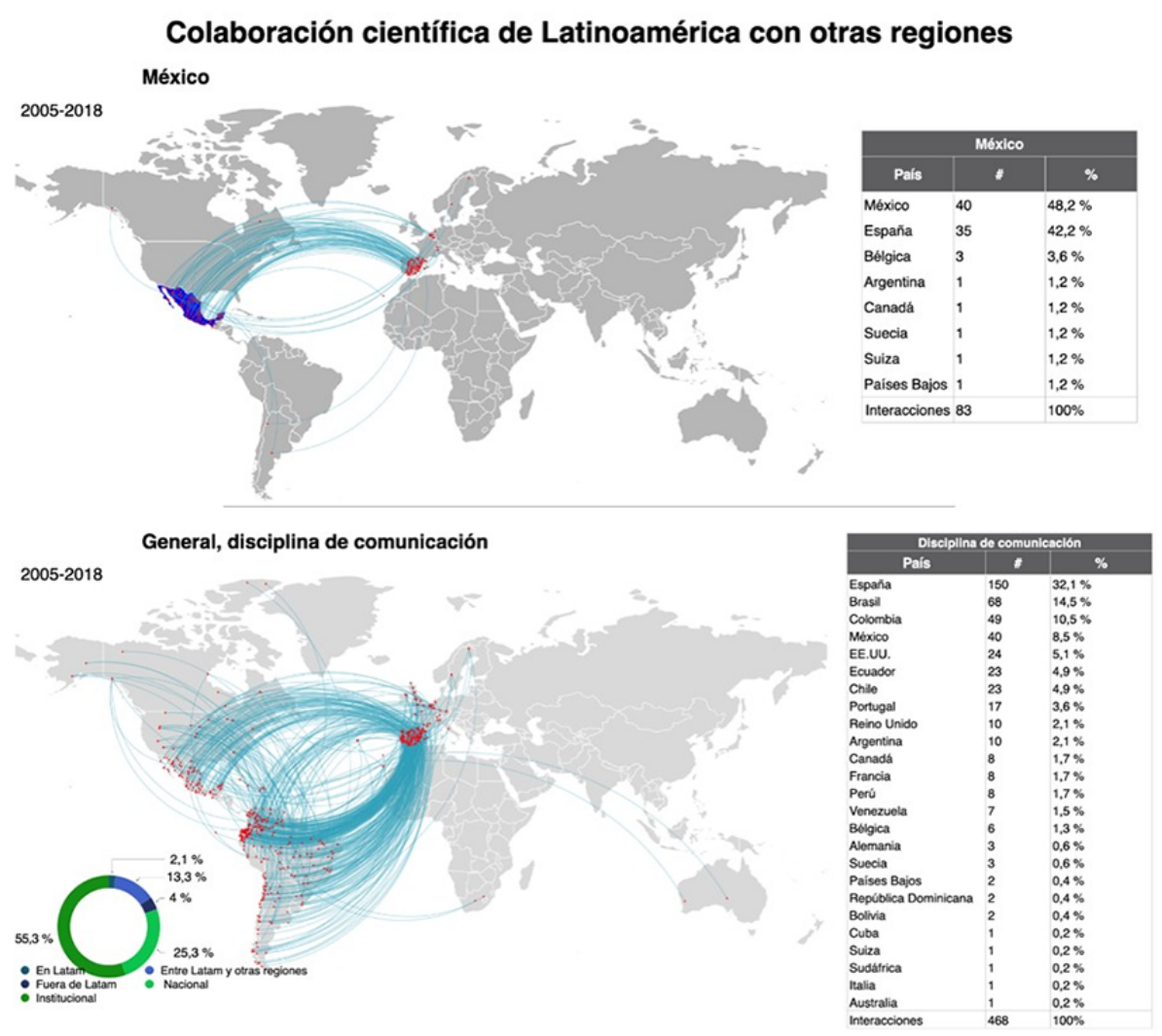

FIGURA 11

Redes de colaboración científica entre Latinoamérica y otras regiones, disciplina de Comunicación, 2005-2018 Fuente: elaboración propia a partir de datos de Redalyc, UAEM (2020).

En las redes de colaboración de países no latinoamericanos (el segundo tipo de colaboración más frecuente) se observa la participación de académicos adscritos a 22 países, donde España se muestra como el país más dinámico, mientras que en segundo lugar se encuentra el Reino Unido. En las redes de colaboración fuera de Latinoamérica se observa una participación diversificada entre las regiones de Europa, Norteamérica, África, Asia y Australia (Figura 12). 
General, disciplina de comunicación

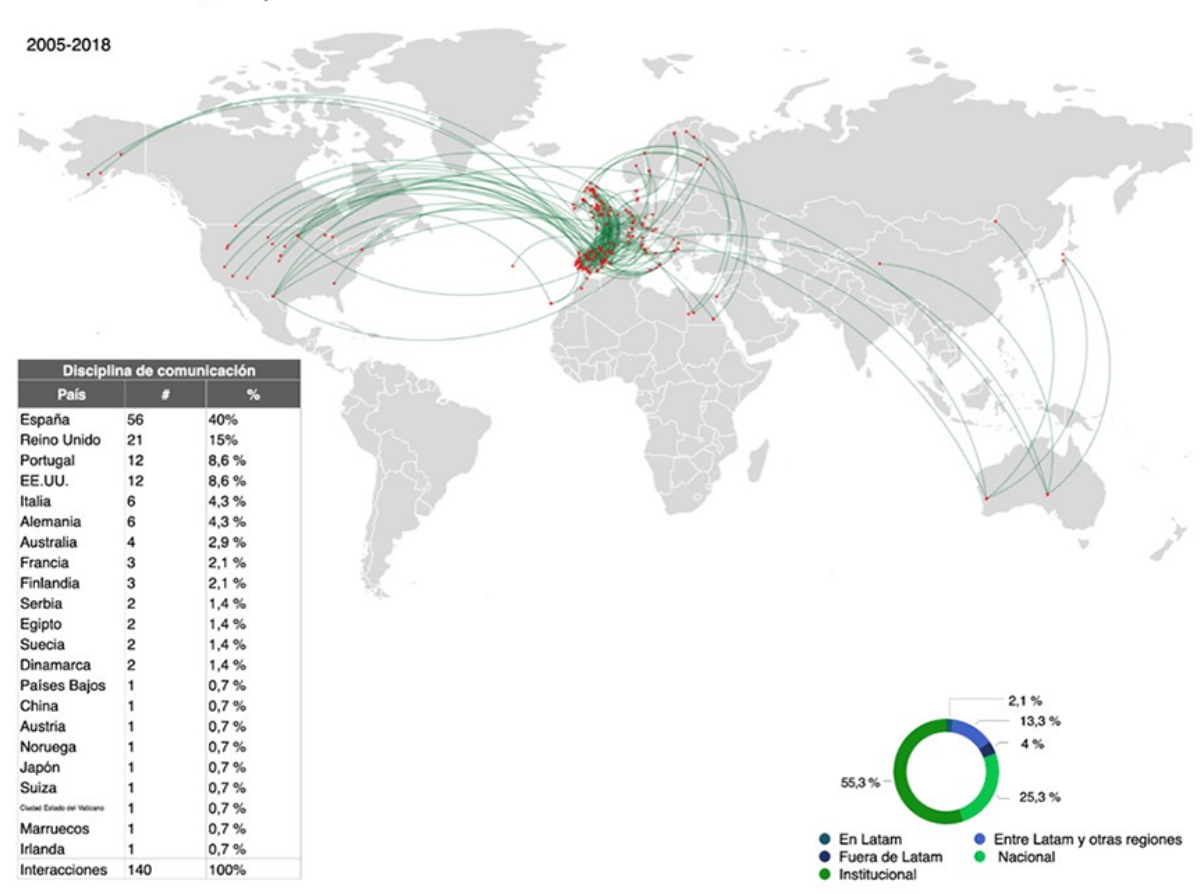

FIGURA 12.

Redes de colaboración científica fuera de Latinoamérica

Fuente: elaboración propia a partir de datos de Redalyc, UAEM (2020).

Finalmente, se registran 302 redes de colaboración institucional en la producción científica de la disciplina de Comunicación, generada en Iberoamérica. Como se observa en la Figura 13, el comportamiento por país es diverso. Existen comunidades más dinámicas en este aspecto, como son los casos de España y Brasil, países donde, respectivamente, 80 instituciones han construido redes de colaboración institucional, y que han generado, respectivamente, 791 y 478 interacciones bajo este esquema de trabajo. En contraste, casos como el de Perú, que registra la participación de cuatro instituciones que han producido 20 interacciones en colaboración institucional. De esta forma, puede observarse la diversidad y alcance de las interacciones que dan lugar a la generación de conocimiento, bajo comportamientos heterogéneos por comunidad científica (regiones, países, instituciones) que, en su diversidad, componen una narrativa científica. 

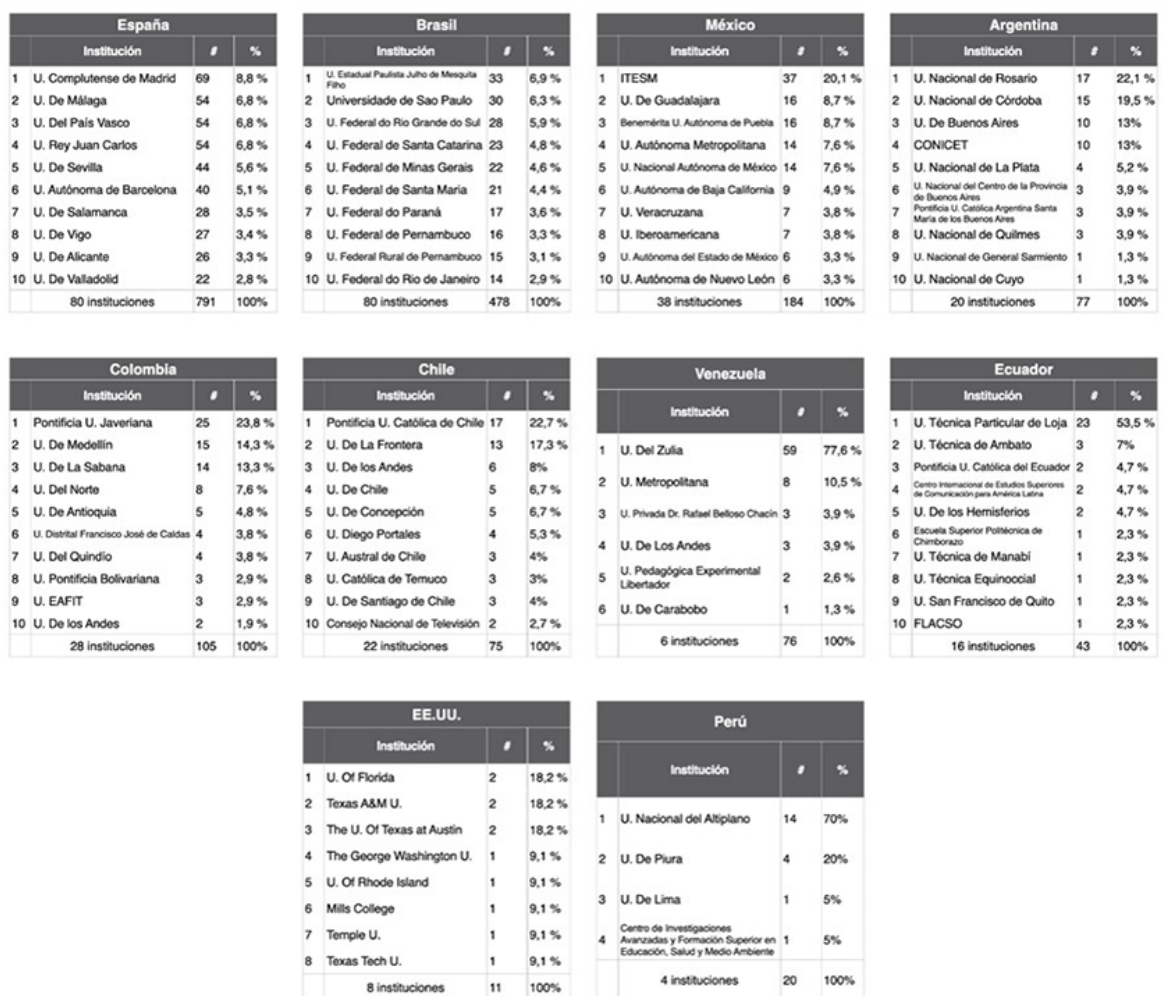

FIGURA 13

Redes de colaboración científica institucional en Latinoamérica

Fuente: elaboración propia a partir de datos de Redalyc, UAEM (2020)

\section{La trayectoria de la comunicación en Iberoamérica}

Los estudios bibliométricos de la literatura científica de la disciplina de comunicación en Iberoamérica, generalmente se enfocan en los indicadores de impacto, los cuales son de estricto carácter cuantitativo y basados en características indirectas a la labor editorial de las revistas científicas, como la citación, lo que no permite dar cuenta de las dinámicas de publicación de las comunidades científicas ni de la tendencias en la generación de conocimiento por parte de los investigadores, sino que muestran únicamente la recepción por otros autores por medio de las citas. Asimismo, los trabajos existentes acerca de la disciplina toman como punto de partida universos poco representativos para regiones como Latinoamérica o Iberoamérica. Ejemplo de lo anterior son los trabajos realizados por Delgado-López-Cózar y Repiso-Caballero (2013), Piedra (2010), Méndez-Rátiva y Gregorio-Chaviano (2014), Castillo-Esparcia et al. (2012); Bravo-Vinaja y Sanz-Casado (2008) y Castillo-Pérez et al. (2015).

A partir de la Segunda Guerra Mundial, la publicación en revistas científicas se consolidó en el principal indicador y medio de consolidación de las disciplinas científicas, dado que permitió la difusión e interpelación del conocimiento científico a escala global. No obstante, también se constituyeron y legitimaron canales de circulación como "centrales" e "idóneos", en la medida en que el concepto de calidad del conocimiento científico fue reemplazado por el de excelencia, a su vez asociado con la publicación en revistas científicas incluidas, por ejemplo, en el Science Citation Index, creado en la década de los 60 por Eugene Garfield (Guédon, 2008). De esta forma, se privilegió, a partir de entonces, la publicación en revistas incluidas en 
bases de datos del "mainstream" (Web of Science y Scopus), en detrimento de los canales de comunicación no incluidos en estos universos.

Estas dinámicas de caracterización del desempeño académico han aportado a la legitimación de un sistema competitivo organizado en rankings, a su vez estructurados a partir del Factor de Impacto, pese a la extensa documentación de sus límites y debilidades, incluso metodológicos (Guédon et al., 2019). Se trata de un escenario institucionalizado a partir de los esquemas de evaluación al desempeño de los investigadores, los cuales toman como punto de referencia indicadores cienciométricos basados en modelos propios del norte global, que no necesariamente reflejan el desarrollo intelectual de los diferentes campos científicos. En el caso de Latinoamérica, la presión por tener presencia en tales rankings y encajar en tales cánones, ha propiciado un modelo que sigue la premisa "publicar globalmente y perecer localmente" vs. "perecer globalmente y publicar localmente" (Beigel, 2013; Fanelli \& Larivière, 2016; Naidorf \& Perrotta, 2017).

Derivado de dicho paradigma de evaluación y comunicación de la ciencia, uno de los principales conflictos que se observa en la dinámica científica latinoamericana, es la dependencia académica a las tendencias de medición y desarrollo científico de los países que dan origen a la ciencia de corriente principal y su influencia sobre las instituciones que miden el desempeño de revistas y autores, lo cual origina un modelo de centroperiferia en el que se le da preferencia a citar artículos de las dos principales regiones, Norteamérica y Europa, restando visibilidad a la producción latinoamericana (Mosbah-Natanson \& Gingras, 2013). De tal forma, el ámbito científico se enfrenta a un panorama donde la evaluación al investigador y las prácticas de los propios académicos se orientan por un paradigma que valora circuitos de comunicación específicos, con agendas de investigación puntuales y en idiomas determinados; estos a su vez evaluados a partir de métricas cuyo carácter no permite dar cuenta de forma integral de la manera en que una disciplina está siendo construida, fuera de dónde está siendo citada o del Factor de Impacto o Índice H que muestran las revistas donde las aportaciones científicas han sido publicadas.

Desde otra perspectiva y metodología, el presente análisis expuso otra propuesta metodológica para reconstruir una cartografía de la disciplina de comunicación, a partir de la producción científica publicada en un modelo no comercial de publicación en acceso abierto, en las 27 revistas de la disciplina integradas a Redalyc, donde Iberoamérica, y principalmente Latinoamérica, tiene una alta representatividad. El estudio permitió reconstruir el escenario general de la disciplina y de las principales comunidades científicas nacionales que participan en el campo de conocimiento de la comunicación, identificando a los actores que con el paso del tiempo han participado en su narrativa, los canales de comunicación mediante los cuales se está construyendo permanentemente, las dinámicas de publicación de los diversos actores y las redes de colaboración que se han articulado en la generación de conocimiento.

La actividad de los diferentes campos científicos se concibe como una actividad en permanente transformación, a partir de la interpelación entre actores que, desde diferentes perspectivas analíticas y procederes metodológicos, construyen una narrativa que tiene lugar a partir de la publicación en revistas científicas. En el presente estudio, se partió de una perspectiva que considera a la comunicación científica como una actividad valiosa en sí misma que puede conceptualizarse a partir de las dinámicas de publicación y colaboración, y que brinda la posibilidad de construir una cartografía compleja de los hábitos y dinámicas a partir de los cuales se constituyen los diferentes campos científicos, independientemente del Factor de Impacto o posición en un ranking con que cuente una revista científica. De esta forma, el presente análisis constituye una perspectiva complementaria a los trabajos realizados sobre la trayectoria de la disciplina en México, los cuales, desde una perspectiva cualitativa, han identificado la forma en que la disciplina se institucionalizó en México y en Latinoamérica como un campo formal de conocimiento. 


\section{Conclusiones}

El análisis de la producción científica de la disciplina de comunicación, publicada en acceso abierto, permitió identificar que en la narrativa disciplinar han aportado 65 países, lo que denota la diversidad de actores que se han sumado a la "gran conversación" en el curso de 14 años. En la conversación es concluyente la centralidad de España, Brasil y México en la aportación de literatura científica, siendo también las comunidades con mayor potencial de diálogo internacional mediante la publicación en el extranjero, a los que se suma Argentina. Se identificó a México, España y Ecuador como los principales países editores internacionales en la disciplina, así como la especial fuerza de las redes de colaboración entre Latinoamérica y España, incluso superior a las externas entre países latinoamericanos. La cartografía esbozada invita a reflexionar sobre la forma en que se concibe a los campos de conocimiento científico en la actualidad: ¿Se les concibe como conversaciones permanentes que buscan incidir, directa o indirectamente, en las problemáticas locales, regionales y globales? Si es así, ¿En qué medida los análisis basados en citación y en la identificación de si el conocimiento circula en bases de datos del mainstream permite trazar la trayectoria histórica de las diversas disciplinas científicas?

\section{Referencias}

Aguado-López, E., y Becerril-García, A. (3 de junio de 2019). El 15 de agosto se lanza R2020: modelo de publicación abierto, propiedad de la academia, sin fines de lucro, sustentable, con métricas responsables y no subordinado. Periscopio Redalyc. https://www.redalyc.org/redalyc/periscopio/redalyc2020.html

Aguado-López, E., Becerril-García, A., y Godínez-Larios, S. (2018). Asociarse o perecer: la colaboración funcional en las ciencias sociales latinoamericanas. Revista Española de Investigaciones Sociológicas, 161, 3-22. https://doi.or $\mathrm{g} / 10.5477 /$ cis/reis. 161.3

Aguado-López, E., Becerril-García, A., Rogel-Salazar, R., Garduño-Oropeza, G., Zúñiga-Roca, M. F., Babini, D., López-López, W., y Melero, R. (2013). Una métrica alternativa y comprehensiva para el análisis de la actividad cientifica: la metodología Redalyc-Fractal(n. ${ }^{\circ}$ 2, pp. 1-13). Laboratorio de Cienciometría RedalycFractal/Redalyc-UAEM. http://ri.uaemex.mx/bitstream/handle/20.500.11799/242/MetodologiaRedalycFrac tal_Aguado_Becerril.pdf?sequence $=3 \&$ isAllowed $=y$

Beigel, F. (2013). Centros y periferias en la circulación internacional del conocimiento. Nueva Sociedad, 245, 110-123. https://nuso.org/media/articles/downloads/3944_1.pdf

Bravo-Vinaja, A., y Sanz-Casado, E. (2008). Análisis bibliométrico de la producción científica de México en ciencias agrícolas durante el periodo 1983-2002. Revista Fitotecnia Mexicana, 31(3), 187-194. https://www.redalyc.org /comocitar.oa?id=61031301

Castillo-Esparcia, A., Rubio-Moraga, Á., y Almansa-Martínez, A. (2012). La investigación en comunicación. Análisis bibliométrico de las revistas de mayor impacto del ISI. Revista Latina de Comunicación Social, 67, 248-270. htt ps://doi.org/10.4185/RLCS-067-955-248-270

Castillo-Pérez, J. J., Muñoz-Valera, L., García-Gómez, F., y Mejía-Aranguré, J. M. (2015). Análisis bibliométrico de la producción científica sobre la influenza en México, 2000-2012. Revista Médica del Instituto Mexicano del Seguro Social, 53(3), 294-301. https://www.medigraphic.com/cgi-bin/new/resumen.cgi?IDARTICULO=58486

Craig, R. T. (1999). Communication theory as a field. Communication Theory, 9(2), 119-161. https://doi.org/10.11 11/j.1468-2885.1999.tb00355.x

Craig, R. T. (2013). Communication in the conversation of disciplines. Russian Journal of Communication, 1(1), 7-23. https://doi.org/10.1080/19409419.2008.10756694

Delgado-López-Cózar, E., y Repiso-Caballero, R. (2013). El impacto de las revistas de comunicación: Comparando Google Scholar Metrics, Web of Science y Scopus. Comunicar, 21(41), 45-52. https://doi.org/10.3916/C41-2 013-04 
Fanelli, D., y Larivière, V. (2016). Researchers' individual publication rate has not increased in a century. PLoS ONE, 11(3). https://doi.org/10.1371/journal.pone.0149504

Fuentes-Navarro, R. (2010a). Investigación de la comunicación: referentes y condiciones internacionales de un diálogo transversal de saberes. Signo y Pensamiento, 29(57), 38-48. https://doi.org/10.11144/Javeriana.syp29-57.inco

Fuentes-Navarro, R. (2010b). Medio siglo del estudio universitario de la comunicación en México: el riesgo del inmediatismo superficial. En Á. M. Ortiz-Marín (Coord.), Anuario CONEICC de Investigación de la Comunicación XVII (pp. 99-115). Consejo Nacional para la Enseñanza y la Investigación de las Ciencias de la Comunicación. https://rei.iteso.mx/bitstream/handle/11117/2661/5805.pdf?sequence=2\&isAllowed=y

González Alcaide, G., y Gómez Ferri, J. (2014). La colaboración científica: principales líneas de investigación y retos de futuro. Revista Española de Documentación Cientifica, 34(4), 1-5. https://doi.org/10.3989/redc.2014.4.1186

Guédon, J. -C. (2008). Open access and the divide between "mainstream" and "peripheral" science. E-LIS, 1-25. http ://eprints.rclis.org/10778/1/Brazil-final.pdf

Guédon, J. -C. (2017). Open access: Toward the internet of the mind. Budapest open access initiative 15 years statement. https://www.budapestopenaccessinitiative.org/open-access-toward-the-internet-of-the-mind

Guédon, J. -C., Jubb, M., Kramer, B., Laakso, M., Schmidt, B., Šimukovič, E., Hansen, J., Kiley, R., Kitson, A., Van der Stelt, W., Markram, K., y Patterson, M. (2019). Future of scholarly publishing and scholarly communication (Informe del Grupo de Expertos de la Comisión Europea). Oficina de Publicaciones de la Unión Europea. htt ps://doi.org/10.2777/836532

López Castañares, R., Dutrénit Bielous, G., Tinoco García, I., y Aguado-López, E. (2013). Informe sobre la producción cientifica de México en revistas iberoamericanas de acceso abierto en redalyc.org 2005-2011. Asociación Nacional de Universidades e Instituciones de Educación Superior/Foro Consultivo Científico y Tecnológico/ International Network for the Availability of Scientific Publications/Universidad Autónoma del Estado de México. http://ri.uaemex.mx/bitstream/handle/20.500.11799/240/InformeMexico_Lopez_Dutrenit.pdf?seq uence $=3 \&$ is Allowed $=\mathrm{y}$

Méndez-Rátiva, C. P., y Gregorio-Chaviano, O. (2014). Aproximación a la comunicación desde la perspectiva teórica y bibliométrica. Un análisis en Web of Science 2008-2012. Signo y Pensamiento, 32(64), 114-135. https://doi. org/10.11144/Javeriana.SyP33-64.actb

Mosbah-Natanson, S., y Gingras, Y. (2013). The globalization of social sciences? Evidence from quantitative analysis of 30 years of production, collaboration and citations in the social sciences (1980-2009). Current Sociology, 62(5), 626-646. https://doi.org/10.1177/0011392113498866

Naidorf, J., y Perrotta, D. (2017). La privatización del acceso abierto. Nuevas formas de colonización académica en América Latina y su impacto en la evaluación de la investigación. Universidades, 73, 41-50. https://www.redal yc.org/pdf/373/37353384005.pdf

Pereira, J. M. (2005). La comunicación: un campo de conocimiento en construcción Reflexiones sobre la comunicación social en Colombia. Investigación \& Desarrollo, 13(2), 412-441. http://rcientificas.uninorte.edu.co/index.php /investigacion/index

Piedra, Y. (2010). Campo científico de la comunicación: examinando su estructura intelectual a través del análisis de cocitación. Revista Latina de Comunicación Social, 65, 204-213. https://doi.org/10.4185/RLCS-65-2010-893 $-204-213$

Rodríguez, L. (2010, abril). Las revistas iberoamericanas en Web of Science y Scopus: visibilidad internacional e indicadores de calidad. Presentado en VII Seminario Hispano-Mexicano de Investigación en Bibliotecología y Documentación, Ciudad de México. http://eprints.rclis.org/14490/1/LuisRY7Encuentro.pdf

Russell, J. M., Ainsworth, S., del Río, J. A., Narváez-Berthelemot, N., y Cortés, H. D. (2007). Colaboración científica entre países de la región latinoamericana. Revista Española de Documentación Científica, 30(2), 180-198. http:/ /redc.revistas.csic.es/index.php/redc/article/viewFile/378/390

SCImago Journal \& Country Rank. (2019). Rankings. SJR. https://www.scimagojr.com 
Soto del Ángel, J. (2008). La autorreproducción del sistema de la ciencia en el campo académico de la comunicación en México. Una reflexión a partir de Niklas Lubmann (Tesis de doctorado inédita). Universidad Veracruzana, México.

\section{Notas}

* Artículo de investigación científica. Este artículo es producto de un seminario de investigación de cuatro semestres para la obtención de grado de licenciatura de Daniel Francisco Flores García, con la asesoría de investigación de la Dra. Arianna Becerril García.

[1] Las 27 revistas son: Razón y Palabra, México-Ecuador; Comunicar, España; Revista FAMECOS: mídia, cultura e tecnologia, Brasil; Revista Latina de Comunicación Social, España; Vivat Academia, España; Chasqui. Revista Latinoamericana de Comunicación, Ecuador; ICONO 14, Revista de comunicación y tecnologías emergentes, España; Palabra Clave, Colombia; Galáxia, Brasil; Signo y Pensamiento, Colombia; Intercom-Revista Brasileira de Ciências da Comunicação, Brasil; Ámbitos. Revista Internacional de Comunicación, España; Matrizes, Brasil; Comunicación y Sociedad, México; Revista de Comunicación de la SEECI, España; La Trama de la Comunicación, Argentina; Anagramas Rumbos y Sentidos de la Comunicación, Colombia; Interin, Brasil; Global Media Journal, México; Quórum Académico, Venezuela; Comunicación y Hombre, España; Cuadernos de Información, Chile; Sphera Pública, España; ModaPalavra e-periódico, Brasil; Punto Cero. Universidad Católica Boliviana, Bolivia; Mètode Science Studies Journal, España; COMUNI@CCION: Revista de Investigación en Comunicación y Desarrollo, Perú.

[2] Por formas de autor se hace referencia a la cantidad de autorías registradas en la producción científica. A diferencia de los datos de país e institución, esta variable no se normaliza, sino que el conteo se realiza a partir de la información proporcionada por los propios autores. Por ejemplo, un autor cuyo nombre es Luis Gómez Méndez, puede tener las firmas Luis G. Méndez o Luis Gómez-Méndez, en tal caso, se consideran dos firmas de autor.

\section{Licencia Creative Commons CC BY 4.0}

Para citar este artículo: Flores-García, D., y Becerril-García, A. (2020). La comunicación en México: un análisis de la producción científica disciplinaria en el contexto iberoamericano. Signo y Pensamiento, 39. ht tps://doi.org/10.11144/Javeriana.syp39.cmap 